Currículo sem Fronteiras, v. 19, n. 3, p. 1175-1187, set./dez. 2019

\title{
INQUIETAÇÕES SOBRE A EDUCAÇÃO INCLUSIVA NO SUL GLOBAL - Um ponto de vista histórico-cultural ${ }^{1}$
}

\author{
Alfredo J. Artiles \\ Arizona State University, Estados Unidos
}

\begin{abstract}
Resumo
O movimento pela educação inclusiva tornou-se um fenômeno global. Este movimento trouxe muita atenção necessária na esfera da política e do desenvolvimento internacional. No entanto, limitações foram consistentemente identificadas na literatura ao longo do tempo. Eu identifico três limitações de grande importância, a saber, a ambiguidade conceitual, o estreitamento das populações-alvo e os limites no desenho e implementação dos estudos sobre a inclusão. Pretendo aprofundar essa crítica usando uma perspectiva histórico-cultural, discutindo os limites das políticas de inclusão como uma solução técnica, a confiança depositada em suposições que criam pontos cegos e riscos para a implementação da educação inclusiva e a falta de atenção às barreiras estruturais e estratificação que impedem o desenvolvimento de programas de inclusão efetivos. Concluo com uma discussão de recomendações para futuras pesquisas sobre educação inclusiva.
\end{abstract}

Palavras-chave: educação inclusiva; Sul Global; perspectiva histórico-cultural.

\begin{abstract}
Inclusive education has become a global phenomenon. This movement has brought much-needed attention in the policy and international development realms. However, shortcomings have been consistently identified in this literature over time. I identify briefly three such limitations, namely conceptual ambiguity, a narrowing of target populations, and limits in the design and implementation of studies. I aim to deepen this critique using a cultural historical perspective by discussing the limits of inclusion as a technical solution, the reliance on assumptions that create blind spots and risks for the implementation of inclusive education and the lack of attention to structural barriers and stratification that hinder the impact of inclusion. I conclude with a discussion of recommendations for future inclusive education research.
\end{abstract}

Keywords: inclusive education; Global South; cultural historical perspective.

ISSN 1645-1384 (online) www.curriculosemfronteiras.org

http://dx.doi.org/10.35786/1645-1384.v19.n3.22 
Cerca de 25 anos atrás, a educação inclusiva ${ }^{2}$ ganhou visibilidade internacional através de vários acordos multinacionais, programas de políticas e relatórios, incluindo a Declaração de Salamanca, a Convenção das Nações Unidas sobre os Direitos das Pessoas com Deficiência, o Relatório Mundial sobre Deficiência (OMS/Banco Mundial, 2011) e os Objetivos de Desenvolvimento da Sustentabilidade da Organização das Nações Unidas (ONU). Grupos ativos nas nações do Norte Global ${ }^{4}$ desempenharam um papel central na criação desse movimento, mas as notícias sobre educação inclusiva se espalharam rapidamente para o Sul Global. O escopo deste projeto era ambicioso - tornar a educação acessível a todos os grupos de alunos, independentemente de suas origens e necessidades, mas com o compromisso de incluir os grupos mais vulneráveis devido a gênero, raça, etnia, idioma, nível de habilidade, origem nacional, religião, classe social ou qualquer outra classificação. Igualmente importante, o movimento pela inclusão prometeu a esses grupos de estudantes uma participação significativa em programas educacionais e resultados equitativos entre os grupos. Grandes reformas foram realizadas e muitos estudantes se beneficiaram.

No entanto, questões e desafios inquietantes surgiram. Por exemplo, a atenção e interesse sobre este tópico na comunidade de pesquisa murchou, conforme refletido no número decrescente de publicações sobre esse tema nos Estados Unidos da América e no Reino Unido. Além disso, o envolvimento com a inclusão no setor de desenvolvimento tem sido lento e problemático, criando desafios conceituais e de implementação, particularmente em contextos do Sul Global (GRECH, 2016). Como vou elaborar em uma seção subsequente, esses desafios se relacionam com a natureza do conhecimento usado em cenários do Sul Global, questões sobre as perspectivas usadas e os reconhecimentos (errôneos) e representações (errôneas) de deficiência que são legitimados nestes esforços. Em relação ao campo da educação, três problemas foram consistentemente identificados (ARTILES \& KOZLESKI, 2016; KOZLESKI, ARTILES \& WAITOLLER, 2014):

1. Falta de clareza conceitual. A educação inclusiva é uma ideia em busca de significado. As revisões de literatura sugerem que a inclusão tende a ser definida apenas como colocação de alunos em salas de aula educacionais em geral, em vez da transformação de sistemas educacionais. Definições claras estão faltando em relatórios, já que em alguns casos a inclusão pode ser equiparada à colocação em programas separados dentro das escolas comuns. Nesse sentido, levantou-se a questão de saber se estamos presenciando o surgimento de exclusões inclusivas (ARTILES, 2011; CARBADO, FISK \& GULATI, 2008) 5 .

2. Todos significa certos grupos. A literatura sobre inclusão evoluiu do objetivo de servir todos os alunos para se concentrar nos alunos com deficiências. Nesse sentido, a educação inclusiva tornou-se a nova educação especial. Alguns estudiosos diferenciam sistemas de inclusão dos sistemas de educação especial e defendem a coexistência efetiva de ambos sistemas (FLORIAN, 2019). O estreitamento das populações constitui uma contradição significativa nessa literatura que aspirava abranger todas as formas de diversidade. 
3. Pesquisa limitada. A pesquisa em educação inclusiva tem sido amplamente produzida na América do Norte e na Europa Ocidental. O limitado escopo geográfico desta base de conhecimento se traduz em ideias e princípios que são mais relevantes para certos contextos nacionais e culturais específicos. Limitações teóricas e metodológicas foram identificadas como por exemplo, pouca atenção tem sido dada às condições dos sistemas educacionais nos quais os alunos são incluídos. Muitos estudos carecem de detalhes sobre aspectos essenciais como a demografia de amostras e as características do ambiente. É significativo que os resultados dos alunos tenham sido raramente documentados nesses estudos (ARTILES, KOZLESKI, DORN e CHRISTENSEN, 2006).

O objetivo deste artigo é duplo. Em primeiro lugar, aprofundo a crítica dos estudos sobre educação inclusiva com ênfase particular nos contextos do Sul Global. Em segundo lugar, utilizo uma perspectiva histórico-cultural para delinear quatro implicações para futuras pesquisas sobre inclusão.

\section{Os limites do universalismo: educação inclusiva nos mundos de diferença}

Há pelo menos três limitações significativas nesta literatura que erodem ainda mais o impacto e as contribuições da educação inclusiva, particularmente no Sul global. Estas incluem o privilégio de uma perspectiva técnica, a natureza centrada no Ocidente da inclusão e a ausência de imaginação interdisciplinar para explicar as dimensões culturais, espaciais e históricas da inclusão.

\section{Inclusão como solução técnica}

Uma limitação importante da pesquisa sobre a política e prática da educação inclusiva é o enfoque que prioriza soluções técnicas para um problema muito complexo. Esta literatura é fundamentada em uma visão de justiça social, a saber, expandir o acesso educacional, a participação e os resultados para grupos vulneráveis. No centro deste trabalho está a transformação de políticas, a preparação de pessoal para ensinar uma população estudantil cada vez mais diversificada na educação geral e o fortalecimento da capacidade dos sistemas escolares de absorver grupos sociais tradicionalmente excluídos. No entanto, essas ideias sobre a inclusão viajam para o Sul Global como inovações técnicas que prometem modernizar sistemas educacionais geralmente subdesenvolvidos. Programas para preparar educadores para a complexa e crescente heterogeneidade das necessidades dos estudantes em todo o mundo são escassos, mas muitos líderes de governo no Sul Global adotam políticas de inclusão com a ilusão de que a modernização seguirá. Essa prática se torna uma forma de deferência judicial (EDELMAN \& TALESH, 2011) - a suposição de que a mera presença de políticas significa que as escolas cumprem a lei e atendem às necessidades de inclusão da população estudantil.

Nesta lógica, a importação de ideias e tecnologias do Norte Global sinaliza progresso. Por exemplo, a adoção de modelos como inclusão completa, Sistemas de Suporte Níveis 
Múltiplos ${ }^{6}$ e Resposta à Intervenção comunica que as nações do Sul estão avançando as agendas de inclusão, cumprindo as exigências de desenvolvimento das agências internacionais para aumentar a cobertura e atender expectativas de qualidade. Mas a infusão dessas inovações nos sistemas escolares é impulsionada pelo objetivo de aumentar a precisão do diagnóstico, ou seja, alcançar maior diferenciação na identificação dos tipos de aprendizes é equiparado à sofisticação científica e ao aumento da qualidade escolar. No entanto, como explico nas próximas duas seções, os defensores da inclusão em todo o mundo devem estar cientes da natureza injusta das sociedades e dos sistemas escolares que podem estar prejudicando o desempenho escolar dos alunos. Por exemplo, as taxas de insucesso escolar observadas no Sul Global (por exemplo, baixo desempenho acadêmico, reprovações e taxas de evasão escolar) podem ser um artefato da falta de capacidade dos sistemas e da onipresença de baixas oportunidades educacionais. Uma vez que essas influências em potencial sejam reconhecidas, os defensores da educação inclusiva devem se perguntar: quais são os alunos vulneráveis incluídos? Essa reflexividade nos permitirá evitar transformar a inclusão em um projeto assimilacionista. De fato, forjar sistemas de educação inclusivos requer atenção ao papel da história, do poder e dos contextos socioculturais.

\section{Limitações e riscos de uma ideia centrada na localização}

Há suposições enganosas e considerações críticas que muitos defensores e pesquisadores da inclusão ignoram, criando assim pontos cegos e exacerbando os riscos que têm repercussões significativas para o futuro da educação inclusiva. Eu aludi brevemente a suposições sobre o universalismo, a falta de atenção aos legados coloniais e uma consciência insuficiente sobre as maneiras pelas quais diferentes significados de diferença e inclusão entre os contextos moldam a implementação da inclusão.

Em primeiro lugar, a educação inclusiva foi articulada e estudada com o pressuposto de que a visão dominante da inclusão na América do Norte e na Europa Ocidental tem um valor universal. Em alguns aspectos, existem valores e princípios de educação que são relevantes para todos em todo o mundo, como a importância do reconhecimento e redistribuição de recursos por parte dos grupos, o valor da participação significativa, a necessidade de igualdade de resultados e a necessidade de autorrepresentação. Lembremo-nos, no entanto, que a pesquisa sobre educação inclusiva viajou por localidades globais, às vezes como parte das agendas de desenvolvimento de organizações internacionais. Esses esforços muitas vezes ignoraram a variações substanciais na organização, nas infraestruturas de desenvolvimento e na qualidade dos sistemas educacionais em todo o mundo, particularmente no Sul Global. A desconsideração de contingências contextuais tem consequências importantes para agendas inclusivas.

Um exemplo é a inclusão pelo Norte Global da promoção de uma agenda de reconhecimento usando um discurso de direitos e a pressão pela redistribuição de recursos, abrindo portas de educação geral para indivíduos com deficiências. No entanto, a noção de direitos e os reconhecimentos que ela oferece podem ser desempenhados de forma diferente no Sul Global. Na Guatemala, por exemplo, embora alguns grupos de alunos com 
deficiências tivessem sido atendidos por décadas, somente no final dos anos 2000 foi aprovado um conjunto progressivo de políticas de educação inclusiva. Esse desenvolvimento de políticas sem precedentes ocorreu no contexto de um projeto nacional de democratização após um longo período de conflito militar e opressão de grupos indígenas. Assim, direitos sociais e direitos educacionais foram legalizados para alunos com deficiências. De acordo com o modelo universalista de educação inclusiva do Norte Global, a criação de uma estrutura de direitos garante o acesso e marca a gênese de programas educacionais inclusivos. Mas Caballeros, Artiles, Canto e Perdomo (2016) tornaram visível a natureza fluida dos direitos na Guatemala, uma vez que os alunos com deficiências só podiam acessar programas inclusivos quando os administradores e educadores locais o permitiam. Nesta versão de exclusões inclusivas, o tipo de deficiência influenciou na forma como os alunos eram classificados. Especificamente, as deficiências de baixa incidência (sensorial, física) foram priorizadas em esforços inclusivos, enquanto os alunos com deficiências mais severas (por exemplo, autismo, distúrbios emocionais/comportamentais) ${ }^{7}$ foram considerados como tendo o direito de receber serviços especializados em instalações separadas. Desta forma, a noção de educação inclusiva foi aplicada diferencialmente, por vezes para manter a segregação de certos grupos sem desafiar o quadro de direitos. As agendas de reconhecimento e redistribuição guatemaltecas da educação inclusiva, impulsionadas por um modelo de direitos, apresentavam particularidades que frequentemente estavam situadas nas contingências das comunidades locais. Entendimentos situados similares da educação inclusiva foram documentados na África do Sul, Malaui e Índia (SINGAL \& MUTHUKRISHNA, 2016; WERNING et al., 2016).

Em segundo lugar, a transferência do movimento de inclusão para o Sul global geralmente ignorou o complicado passado do trabalho de desenvolvimento e suas ligações com os legados coloniais. Kennedy e Newton (2016) traçaram as histórias do colonialismo e da deficiência e mostraram como os sistemas econômicos que datam do século XVII reproduziram sistemas exploradores e raciais que tiveram legados duradouros de incapacidade. Outros estudiosos nos lembraram das "desconfortáveis" raízes coloniais do "desenvolvimento" que impuseram condições econômicas neoliberais ao Sul global, às vezes com graves consequências para o avanço da justiça social nessas sociedades (GRECH, 2015). Esse tipo de desenvolvimento geralmente favorece investimentos sociais mínimos e estabelece expectativas econômicas para corpos produtivos que têm consequências nefastas para pessoas com deficiências. Por gerações, as agendas de desenvolvimento do Sul Global foram impostas com atenção mínima aos contextos e histórias locais. Estudiosos criticaram esses legados e argumentaram que "a deficiência permanece nas margens de desenvolvimentos da teoria, da política e da prática” (GRECH \& SOLDATIC, 2016, p. 1). No setor da saúde, por exemplo, a atenção à deficiência é superficial e tende a ser limitada a um trabalho de conscientização ou sensibilização (SWARTZ \& BANTJES, 2016).

Por fim, os formuladores de políticas e os pesquisadores tendem a ignorar como a ideia de inclusão e seus significados de diferença são interpretados em diferentes contextos. O estudo anteriormente citado na Guatemala (CABALLEROS et al., 2016) oferece uma visão interessante. Esses pesquisadores relataram que as pessoas que vivem na pobreza nas áreas 
rurais aspiravam obter inclusão social por meio da educação inclusiva; a promessa desse modelo educacional deu-lhes esperança. A educação inclusiva deu a seus filhos acesso à educação e garantiu-lhes comida através de políticas de merenda escolar. Essa postura foi mais pronunciada entre os pais de crianças com deficiências graves que eram as mais marginalizadas. Por outro lado, esses indivíduos eram ambíguos sobre a educação inclusiva por várias razões. Por exemplo, a promessa de acesso à educação foi dificultada pela violência em ambientes urbanos, dado a dificuldade de caminhar até as escolas em meio à hostilidade das gangues nos bairros. Nas áreas rurais, a caminhada para a escola exacerbou o risco de violência sexual para meninas. Além disso, as pessoas nas áreas rurais enfrentaram barreiras estruturais relacionadas à falta de transporte, tendo em vista a escassez e a localização remota das escolas, particularmente das escolas de ensino fundamental e médio. Os pais das comunidades rurais também questionaram o valor instrumental da educação inclusiva, já que não apenas gastariam mais em transporte, uniformes escolares, etc., mas o retorno do investimento de um diploma de ensino médio seria mínimo, dada a escassez de empregos na região que ofereceria salários mais altos para pessoas com maior nível de escolaridade.

\section{Dos silêncios e da estratificação: para além da inclusão como uma resposta bondosa}

A educação inclusiva nos Estados Unidos tem sido criticada por desconsiderar o papel das forças estratificadoras nos contextos em que este trabalho é implementado. Por exemplo, iniquidades estruturais profundas que afetam as oportunidades educacionais para certos grupos foram ignoradas nessa base de conhecimento, como por exemplo, financiamento escolar e qualidade do professor correlacionados com a raça e a classe social dos estudantes. Isso significa que a literatura de inclusão no Norte global tem sido repleta de ocultamentos teóricos e metodológicos que infundiram sistematicamente silêncios sobre opressão e estratificação para certos grupos de estudantes. O resultado é uma base de conhecimento amplamente daltônica (ARTILES \& KOZLESKI, 2016).

Uma limitação relacionada é que esse corpo de trabalho apagou as interseções de deficiência com outras identidades em questões de pesquisa e métodos de estudo, deixando de documentar a heterogeneidade dessa população e compreender as complexidades socioculturais e históricas das experiências vividas por esses aprendizes (EREVELLES, 2011). Esta é uma omissão crítica considerando o problemático entrelaçamento histórico da deficiência com outras categorias como raça, classe social, idioma e status de imigração (ARTILES, 2011; BAYNTON, 2001). Nesses casos, os estudantes vivem em um vínculo duplo devido às barreiras estruturais e menos oportunidades educacionais impostas não apenas pela deficiência, mas por outras categorias estigmatizantes. Este duplo vínculo foi documentado no Norte Global, refletido na literatura sobre a racialização das deficiências nos Estados Unidos e sobre a superidentificação de estudantes imigrantes como estudantes com deficiências na Espanha, Suécia e Alemanha (ARTILES et al., 2011).

Embora as discussões e estudos de interseccionalidade tendam a se concentrar em dois vetores de diferença de cada vez (por exemplo, incapacidade-raça ou gênero-raça), casos 
recentes tornam palpável a necessidade de investigações intersecionais mais complexas. Considere-se, por exemplo, o processo judicial de ação coletiva Franco-Gonzales x Holder que afetou os detidos no Arizona, Califórnia e Washington. O caso de ação coletiva evoluiu "mais de três anos depois que um processo judicial foi aberto por José Antonio FrancoGonzalez, um imigrante mexicano com deficiência cognitiva que foi detido em instalações federais de imigração por quase cinco anos sem uma audiência ou um advogado” (ACLU, 2013). A Corte decidiu fornecer representação legal a detentos imigrantes com deficiências intelectuais que estavam enfrentando deportação e que não podiam se representar adequadamente em audiências de imigração. Questões de linguagem, status de imigração, etnia e deficiência se entrelaçam nas experiências desses indivíduos e os vetores de diferença que eles incorporam invocam soluções de justiça díspares (talvez contraditórios), isto é, tratamento diferenciado e similar. As formas como membros oficiais de instituições (por exemplo, funcionários escolares, oficiais de imigração) resolvem as mudanças na justiça que são codificadas nas políticas e nas práticas são significativamente inexploradas. Um exemplo, as determinações políticas que excluem as diferenças linguísticas e culturais dos alunos nas considerações sobre diagnóstico da incapacidade de aprendizagem.

Esta evidência emergente confirma a análise histórica de Baynton (2001) mostrando como a deficiência pode ser usada como uma justificativa para discriminar outros grupos marginalizados. Nesse sentido, a deficiência é implantada como "o principal tema da desqualificação" (EREVELLES, 2011, p. 15). Cabe destacar que obteremos uma compreensão mais sutil da interseccionalidade no contexto da educação inclusiva, transcendendo um foco exclusivo em múltiplas categorias demográficas. Por exemplo, a pesquisa no Sul Global pode se concentrar em pessoas com identidades intersetoriais que vivem sob circunstâncias socioculturais específicas, como viver em zonas de conflito e guerra. Mas, como viver nas paisagens sociais e econômicas da guerra interfere nas formas pelas quais a deficiência é reconhecida e representada e como a inclusão é vivenciada? A vulnerabilidade é o único papel disponível para esses indivíduos? Como os vários papéis que as pessoas com deficiência desempenham nessas zonas são reconhecidos e representados? (por exemplo, como combatentes, ativistas, manifestantes). Com quais consequências? (BERGHS \& KABBARA, 2016). Da mesma forma, morar em áreas rurais do Sul Global molda condições de desvantagem para pessoas com deficiência que são qualitativamente diferentes de sofrer de incapacidade em locais urbanos (GARTRELL \& HOBAN, 2016; WERNING et al., 2016). Análises comparativas intersetoriais são necessárias em maior quantidade. Da mesma forma, há uma escassez alarmante de pesquisas sobre "a natureza, a prevalência, o impacto e a prevenção da violência contra as mulheres com deficiência” (GRECH \& SOLDATIC, 2016, p. 2). Essas lacunas de conhecimento infundem silêncios profundamente problemáticos sobre a violência de gênero e a discriminação na implementação de programas inclusivos (DOWSE et al., 2016).

\section{Inclusão no século XXI: um ponto de vista histórico-cultural}


As deficiências da literatura sobre inclusão sugerem que transformações fundamentais são necessárias no conhecimento que os pesquisadores estão produzindo sobre esse tópico crítico e contemporâneo. Eu delineio quatro áreas que precisam de melhorias para futuras investigações sobre educação inclusiva.

\section{O imperativo e as contribuições de uma abordagem situada}

A discussão anterior torna evidente que a inclusão não deve ser tomada como um projeto puramente técnico. Pesquisadores e profissionais devem considerar múltiplas escalas temporais e espaciais ao implementar a inclusão, porque o conhecimento viaja através da mídia cultural. Assim, devemos supor que a inclusão é contextualizada e interpretada de acordo com as histórias locais e as contingências culturais. Além disso, como explicado acima, a noção de inclusão tem bagagem histórica, já que sua categoria central, a deficiência, tem interconexões problemáticas com outras categorias marcadas como raça, gênero, classe social e linguagem. Pesquisas nas ciências sociais e humanas documentaram que essas categorias têm peso estrutural na vida das comunidades e sociedades. Por estas razões, é imperativo que pesquisas futuras dependam de um modelo adaptativo de educação inclusiva que esteja atento às histórias, contextos e necessidades locais. Goodley e Swartz (2016) articularam a necessidade de examinar o nexo global-local como uma estratégia para entender as complexas realidades, prioridades e restrições das agendas de inclusão de incapacidades para que possamos iluminar o lugar da deficiência "onde e quando a deficiência aparece e como (re)emerge geopolítica, temporal e epistemologicamente” (GRECH \& SOLDATIC, 2016, p. 1).

Os pesquisadores devem reconhecer que suas percepções não são o resultado de processos puramente fisiológicos por causa da "inseparabilidade da percepção de todo o conjunto de relações sociais e individuais nas quais ela funciona e das quais é uma expressão”

(WARTOFSKY, 1985, p. 237). Uma implicação chave deste fato é que a pesquisa futura deve considerar os processos interpessoais que estão situados em contextos institucionais. Este ponto de vista nos lembra da urgência de iniciar análises da educação inclusiva, concentrando-se em processos interpessoais, pois é nas interações que os significados da deficiência (entrelaçados com outras categorias sociais) ganham valor.

\section{Imaginação cultural e histórica}

Uma implicação relacionada é que as dimensões culturais e históricas devem ser componentes intrincados da futura pesquisa sobre inclusão (ARTILES, 2019). Não devemos supor que os processos de deficiência e inclusão ocorram em ambientes desprovidos dessas dimensões. Vamos considerar dois conjuntos de descobertas de campos aliados. Primeiro, usando um conjunto de dados representativo nacional, Cherng (2017, p. 180) descobriu recentemente que 
os professores de matemática percebem consistentemente que suas aulas são muito difíceis para os estudantes latinos e negros mesmo depois de controlar os resultados dos testes de matemática, conclusão de tarefas e uma série de outros fatores. Os professores de inglês subestimam as habilidades acadêmicas de estudantes negros e latinos e, em alguns modelos, também de estudantes asiático-americanos.

Por sua vez, a pesquisa experimental em psicologia social sobre a desumanização dos negros "demonstrou que, mesmo controlando o preconceito implícito antinegros, a associação implícita entre negros e macacos pode levar a um endosso maior da violência contra um suspeito negro do que contra um suspeito branco” (GOFF et al., 2008, p. 304).

Quais são as sedimentações históricas desses processos cognitivos em relação aos antigos déficits de raça e deficiência? Como essas tendências perceptivas e preconceitos se desenrolam nos processos interpessoais em sala de aula e na escola? Como essas percepções interferem nas decisões do professor de encaminhar estudantes de cor para educação especial ou aplicar sanções disciplinares? Como Varenne e McDermott (1999, p. 33) nos lembram, "sempre que vemos [identidades raciais ou de deficiência], o mundo institucional que nos faz procurá-las e encontrá-las sempre nos precedeu à cena”.

\section{Avançando as agendas de equidade: a necessidade da consciência legal}

Líderes e profissionais geralmente assumem que as correções de políticas de equidade cuidam das injustiças. Ou seja, a passagem de leis e requisitos legais elimina problemas de equidade. No entanto, como estudos sobre direito, ciência política e educação demonstraram, esse não é o caso. Esse fenômeno foi descrito como deferência legal (EDELMAN \& TALESH, 2011), isto é, a suposição de que a presença de legislação para inclusão educacional se traduz em conformidade. Mas as evidências mostram que estados e secretarias de educação nos Estados Unidos descumprem a legislação existente para reduzir a desproporcionalidade racial na educação especial, manipulando os critérios necessários para desencadear citações legais e ações corretivas (CAVENDISH, ARTILES \& HARRY, 2014; TEFERA \& VOULGARIDES, 2016). Desta forma, os dilemas da diferença são reproduzidos, ou seja, soluções justas que perpetuam as injustiças (ARTILES, 1998). A questão inquietante de Silbey (2005, p. 323) descreve esse fenômeno de uma forma alternativa: "por que as pessoas aceitam um sistema legal que, apesar de suas promessas de tratamento igual, sistematicamente reproduz a desigualdade?”. Pesquisas futuras sobre inclusão devem dar relevância às demandas legais pertinentes aos requisitos de inclusão. Uma abordagem viável é fundamentar o trabalho futuro na noção de conscientização jurídica, ou seja, como os indivíduos interpretam e experimentam leis e políticas em suas práticas cotidianas, às vezes para aplicá-las, combatê-las ou contorná-las (SILBEY, 2005).

\section{Ampliar as possibilidades de representações}


As representações tradicionais da deficiência são enquadradas dentro de uma perspectiva deficitária. De fato, essa população é tipicamente descrita como deficiente e incapaz. Os retratos negativos são compostos quando os alunos com deficiências são de grupos minoritários (estudantes de cor, alunos de baixa renda). Em essência, este é um problema representacional. A comunidade de pesquisa precisa ampliar suas lentes teóricas e analíticas para capturar a engenhosidade dos alunos e suas famílias, independentemente das limitações ou desafios que enfrentam em suas vidas. O ponto de partida deve ser que os seres humanos são protagonistas ativos que se esforçam para melhorar suas condições. A literatura sobre resiliência tem documentado este fato há muitos anos e refinamentos recentes colocam a resiliência em uma unidade de análise sociocultural mais ampla (GUTIERREZ et al., 2017). Meus colegas e eu documentamos como famílias e comunidades desfavorecidas que viviam em terríveis circunstâncias na Guatemala geravam soluções espontâneas para educar seus filhos com necessidades especiais. Por exemplo, mobilizavam recursos de setores fora da educação ou usavam suas redes sociais (CABALLEROS et al., 2016). Os pesquisadores devem procurar construir representações ecologicamente válidas e dignas desses grupos e suas lutas (ARZUBIAGA, ARTILES, KING \& HARRIS-MURRI, 2008; ESPINOZA E VOSSOUGHI, 2014). Finalmente, o que os futuros pesquisadores da área da inclusão deveriam procurar produzir novas formas de representação fundamentadas em "uma ciência empática, uma ciência humanista, capaz de lidar com a potência da narrativa pessoal com serenidade, utilizando as ferramentas da ciência social e da ciência sem que essas ferramentas oprimam a pessoa humana ”(M. Espinoza, comunicação pessoal, 19 de setembro de 2017). 


\section{Notas}

1. Notas do autor: Nota 1) Este artigo é baseado em uma apresentação feita no Seminário Internacional "Que educação para que cidadania? Promessas, perguntas e propostas que interrogam equidade, inclusão e diversidade” realizado na Universidade Federal Rural do Rio de Janeiro (Campus Nova Iguaçu), Rio de Janeiro, Brasil, em setembro de 2018. Agradeço à Professora Sandra Sales por me receber durante esta reunião e por seu apoio durante a preparação deste manuscrito. Nota 2) Agradeço também à Professora Márcia Pletsch por realizar a revisão técnica deste artigo.

2. Embora existam diferenças entre esses termos, eu uso “educação inclusiva” e "inclusão" de forma intercambiável para fins de estilo editorial.

3. Os termos “Norte Global e "Sul Global” invocam "relações geopolíticas de poder" de longa data (DADOS \& CONNELL, 2012, p. 12). Grech (2015, p. 1) explicou que o termo denota "diferenciais de poder, recursos, epistemológicos e outros que, embora não sejam imutáveis, se referem a uma parte substancial do mundo que vive em um cenário de profundas assimetrias geopolíticas, pobreza e isolamento e confrontam centros de concentração de riqueza e de poder acumulados historicamente e perpetuados em tempos de colonialidade. Não existe um Sul Global, mas existem muitos "Suls" globais. Além disso, o Sul Global não está presente apenas, mas também vive no Norte Global”.

4. No Brasil esse fenômeno tem sido chamado por diferentes autores (BUENO et al., 2008; MELLETI e KASSAR, 2013) como a exclusão do interior. Isto é, os alunos com alguma deficiência são matriculados em turmas comuns de ensino, mas excluídos dos processos de escolarização).

5. No Brasil apesar da Política em Educação Especial numa Perspectiva a Inclusiva sugerir como suporte somente o Atendimento Educacional Especializado por meio das salas de recursos multifuncionais, as pesquisas na área tem evidenciado que cada rede de ensino tem implementado suas propostas a partir das suas realidades locais com diferentes suportes, como, por exemplo, o apoio de agentes de inclusão e mediadores em sala de aula, entre outros.

6. Cabe destacar que nos Estados Unidos enquadram-se no público-alvo sujeitos com problemas emocionais e comportamentais, mas que no Brasil segundo a Política Nacional de Educação Especial na Perspectiva da Educação Inclusiva esses sujeitos não se enquadram como público-alvo da educação especial.

\section{Referências}

ACLU (2013). Franco-Gonzalez v. Holder. Disponível em: <https:/www.aclu.org/cases/franco-gonzalez-vholder>. Acesso em 20 abr. 2014.

ARTILES, A.J. The dilemma of difference: Enriching the disproportionality discourse with theory and context. The Journal of Special Education, v. 32, 1998. p. 32-36.

Toward an interdisciplinary understanding of educational equity and difference: The case of the racialization of ability. Educational Researcher, v. 40, 2011. p. 431-445.

. (2019). Re-envisioning equity research: Disability identification disparities as a case in point.

Educational Researcher, August/September/2019. Disponível em

https://journals.sagepub.com/doi/abs/10.3102/0013189X19871949?journalCode=edra. Acesso em 03 out. 2019.

, KOZLESKI, E. B. Inclusive education's promises and trajectories: Critical notes about future research on a venerable idea. Educational Policy Analysis Archives, v. 24, n. 43, 2016. Disponível em: <http://dx.doi.org/10.14507/epaa.24.1919>. Acesso em: 15 jan 2017.

, KOZLESKI, E., WAITOLLER, F. (Eds.). Inclusive education: examining equity on five continents. Cambridge, MA: Harvard Education Press, 2011.

; KOZLESKI, E.; DORN, S.; CHRISTENSEN, C. Learning in inclusive education research: Remediating theory and methods with a transformative agenda. Review of Research in Education, v. 30, 2006. p. 65-108. 
ARZUBIAGA, A.; ARTILES, A.J.; KING, K.; HARRIS-MURRI, N. Beyond research on cultural minorities: Challenges and implications of research as situated cultural practice. Exceptional Children, v. 74, 2008. p. 309-327.

BAYNTON, D. Disability and the justification of inequality in American history. In: LONGMORE, P. K.; UMANSKY, L. (Eds.), The new disability history. NY: NYU Press, 2001. p. 33-57

BERGHS, M.; KABBARA, N. Disabled people in conflicts and wars. In: GRECH, S.; SOLDATIC, K. (Eds.), Disability in the global South: The critical handbook. Cham, Switzerland: Springer, 2016.

BUENO, J. G. S.; MENDES, G. M. L.; SANTOS, R. A. (Orgs.). Deficiência e escolarização: novas perspectivas de análise. Araraquara: Editora Junqueira e Marin, 2008.

CABAlLEROS, M. Z.; ARTILES, A. J.; CANTO, H.; PERDOMO, C. Inclusive education in developing countries: Two case studies from Guatemala. In R. Werning, A. J. Artiles, P. Engelbrecht, M. Z. Caballeros, M. Hummel, \& A. Rothe (Eds.), Keeping the promise? Contextualizing inclusive education in developing countries. Bad Heilbrunn: Klinkhardt, 2016. p. 121-150.

CARBADO, D. W.; FISK, C.; GULATI, G.M. After Inclusion. Duke Law School Public Law \& Legal Theory Paper No. 210; UC Irvine School of Law Research Paper No. 2008-4. 2008. Disponível em: $<$ https://ssrn.com/abstract=1138795. or http://dx.doi.org/10.2139/ssrn.1138795>. Acesso em 21 fev. 2009.

CAVENDISH, W.; ARTILES, A. J.; HARRY, B. Tracking inequality: Does policy legitimize the racialization of disability? Multiple Voices, v.14, n. 2, 2014. p. 30-40.

CHERNG, H.S. If they think I can: Teacher bias and youth of color expectations and achievement. Social Science Research, v. 66, 2017. p. 170-186.

DADOS, N.; CONNELL, R. The global South. Contexts, v. 11, n. 1, 2012. p. 12-13.

DOWSE, L.; FROHMADER, C.; DIDI, A. Violence against disabled women in the global South: Working locally, acting globally. In: GRECH, S.; SOLDATIC, K. (Eds.), Disability in the global South: The critical handbook. Cham, Switzerland: Springer, 2016.

EDELMAN, L.B.; TALESH, S.A. (2011). To comply or not to comply - that isn't the question: How organizations construct the meaning of compliance. In: PARKER, C.; NIELSEN, V.L. (Eds.), Explaining compliance. Northampton, MA: Edward Elgar, 2011. p. 103-122.

EREVELLES, N. Disability and difference in global contexts: Enabling a transformative body politic. New York: Palgrave MacMillan, 2011.

ESPINOZA, M.L., VOSSOUGHI, S. Perceiving learning anew: Social interaction, dignity and educational rights. Harvard Educational Review, v. 84, 2014. p. 285-313.

FLORIAN, L. On the necessary co-existence of special and inclusive education. International Journal of Inclusive Education. 2019. Disponível em: <https://doi.org/10.1080/13603116.2019.1622801>. Acesso em 12 out. 2019.

GARTRELL, A.; HOBAN, E. "Locked in space": Rurality and the politics of location. In: GRECH, S.; SOLDATIC, K. (Eds.). Disability in the global South: The critical handbook. Cham, Switzerland: Springer, 2016.

GOFF, P.A.; EBERHARDT, J.L.; WILLIAMS, M.J.; JACKSON, M.C. Not yet human: Implicit knowledge, historical dehumanization, and contemporary consequences. Journal of Personality and Social Psychology, v. 94, 2008. p. 292-206.

GOODLEY, D.; SWARTZ, L. The place of disability. In: GRECH, S.; SOLDATIC, K. (Eds.), Disability in the global South: The critical handbook. Cham, Switzerland: Springer, 2016.

GRECH, S. Disability and poverty in the global South: Renegotiating development in Guatemala. New York: Palgrave Macmillan, 2015. 
; SOLDATIC, K. Disability in the global South. In: GRECH, S.; SOLDATIC, K. (Eds.), Disability in the global South: The critical handbook. Cham, Switzerland: Springer, 2016.

GUTIERREZ, K.D. et al. Replacing representation with imagination: Finding ingenuity in everyday practices. Review of Research in Education, v. 41, 2017. p. 30-60.

KENNEDY, S.; NEWTON, M.J. The hauntings of slavery: Colonialism and the disabled body in the Caribbean. In: GRECH, S.; SOLDATIC, K. (Eds.), Disability in the global South: The critical handbook. Cham, Switzerland: Springer, 2016.

KOZLESKI, E. B.; ARTILES, A. J.; WAITOLLER, F. (2014). Equity in inclusive education: A cultural historical comparative perspective. In: FLORIAN, L. (Ed.), The Handbook of Special Education. New York: Sage Publications, 2014. p. 231-249.

MELLETI, S. M. F.; KASSAR, M. C. M. (Orgs.). Escolarização de alunos com deficiências: desafios e possibilidades. São Paulo: Mercado das Letras, 2013.

SILBEY, S. S. After legal consciousness. Annual Review of Law and Social Science, v. 1, 2005. p. 323-368.

SINGAL, N.; MUTHUKRISHNA, N. Reflexive re-storying of inclusive education: Evidence from India and South Africa. In: GRECH, S.; SOLDATIC, K. (Eds.), Disability in the global South: The critical handbook. Cham, Switzerland: Springer, 2016.

SWARTZ, L.; BANTJES, J. Disability and global health. In: GRECH, S.; SOLDATIC, K. (Eds.), Disability in the global South: The critical handbook. Cham, Switzerland: Springer, 2016.

TEFERA, A.; VOULGARIDES, C.K. Is educational policy alleviating or perpetuating the racialization of disabilities? Teachers College Record, v. 118, n. 14, 2016. p. 1-24.

VARENNE, H.; MCDERMOTT, R. Successful failure: The school America builds. Boulder, CO Westview Press, 1999.

WARTOFSKY, M.W. Perception, representation and the forms of action: Towards an historical epistemology. In R.S. Cohen \& M.W. Wartofsky (Eds.), A portrait of twenty-five years: Boston colloquium for the philosophy of science 1960-1985. Boston: Reidel, 1985. p. 215-237.

WERNING, R.; ARTILES, A.; ENGELBRECHT, P.; CABALLEROS, M. Z.; HUMMEL, M.; ROTHE, A. (Eds.). Keeping the promise? Contextualizing inclusive education in developing countries. Bad Heilbrunn: Klinkhardt. 2016. Disponível em: <http://www.pedocs.de/frontdoor.php?source_opus= 12353\&la=de>. Acesso em 19 jan. 2017.

WORLD HEALTH ORGANIZATION \& WORLD BANK. World report on disability. Geneva: Switzerland, 2011.

\section{Correspondência}

Alfredo J. Artiles é professor da Arizona State University, Estados Unidos.

E-mail: alfredo.artiles@asu.edu

Texto publicado em Currículo sem Fronteiras com autorização do autor. 